\title{
Selection of hepatitis B surface "escape" mutants during passive immune prophylaxis following liver transplantation: potential impact of genetic changes on polymerase protein function
}

\author{
P L Shields, A Owsianka, W F Carman, E Boxall, S G Hubscher, J Shaw, K O’Donnell, \\ E Elias, D J Mutimer
}

\begin{abstract}
Case report-A patient is described who developed hepatitis B virus (HBV) reinfection five months following liver transplantation. Failure of hepatitis B immunoglobulin prophylaxis was associated with the emergence of mutations. HBV gene sequencing identified nucleotide substitutions associated with amino acid changes, one within the major hydrophilic region (MHR) of the HBV surface antigen at amino acid position 144 and one outside the MHR. Because of the overlapping reading frames of surface and polymerase genes, the latter surface antigen change was associated with an amino acid change in the polymerase protein. The patient developed significant allograft hepatitis and was treated with lamivudine (3TC) $100 \mathrm{mg}$ daily. Rapid decline of serum HBV DNA was observed with loss of HBV e antigen and HBV surface antigen from serum. There was normalisation of liver biochemistry, and liver immunohistochemistry showed a reduction in $\mathrm{HBV}$ core and disappearance of HBs antigen staining.

Conclusion-Surface antigen encoding gene mutations associated with HBIg escape may be associated with alteration of the polymerase protein. The polymerase changes may affect sensitivity to antiviral treatment. Selection pressure on one HBV reading frame (for example, HBIg pressure on HBsAg, or nucleoside analogue pressure on polymerase protein) may alter the gene product of the overlapping frame. Such interactions are relevant to strategies employing passive immune prophylaxis and antiviral treatment. (Gut 1999;45:306-309)
\end{abstract}

Keywords: liver transplantation; prophylaxis; escape mutants; lamivudine

Liver transplantation is now an established treatment for end stage liver disease, and patients with hepatitis $B$ virus (HBV) induced cirrhosis account for a substantial proportion of those referred for liver transplantation. Recurrence of $\mathrm{HBV}$ in the graft has a significant impact on both graft and patient survival. To minimise this risk, hepatitis B immunoglobulin (HBIg) is administered from the time of transplantation. These preparations contain high titres of antibody to the HBV surface antigen (HBsAg). Without prophylaxis, recurrent infection is observed in more than $80 \%$ of transplanted patients. ${ }^{1}$ Despite sustained HBIg prophylaxis, many develop recurrence, with accelerated liver disease. HBIg prophylaxis is most likely to be effective for patients with low levels of viral replication at the time of transplantation. ${ }^{2}$

HBsAg contains a highly antigenic segment known as the major hydrophilic region (MHR) from amino acids 100-169. The MHR consists of a complex and interleaved set of continuous and discontinuous epitopes defined by disulphide bridging. The structure has been recently suggested by phage epitope mapping studies. ${ }^{3}$ The "a determinant" is part of the MHR, and classically includes amino acids 124-147 of the HBsAg. The MHR is the principal binding site of anti-HBs following natural infection, after immunisation, and during HBIg prophylaxis, although areas up and downstream of this may also be important in neutralisation. Mutations may result in HBsAg amino acid substitutions, and are observed in the context of vaccination against $\mathrm{HBV}^{4}$ and during HBIg prophylaxis after liver transplantation. ${ }^{56}$ It has been proposed (though has not been formally proven) that viral "escape" occurs, and significant viral replication may be observed despite high titres of neutralising antibody. The subject of $S$ gene variation has been recently reviewed. ${ }^{7}$

Useful antiviral strategies for the treatment of established graft infection have not been reported. Interferon seems ineffective. Adenine arabinoside monophosphate, ${ }^{8}$ and ganciclovir ${ }^{9}$ may reduce serum HBV DNA during treat-

Abbreviations used in this paper: $\mathrm{HBIg}$, hepatitis B immunoglobulin; MHR, major hydrophilic region; PCR, polymerase chain reaction. 
ment, but these agents are unsuitable for long term use, and relapse occurs when treatment is stopped. Lamivudine has been used in this setting, ${ }^{10-12}$ and seems to be well tolerated. Prompt reduction in serum HBV DNA was observed for all treated patients with small numbers of patients becoming serum $\mathrm{HBsAg}$ negative. ${ }^{13} 14$

We report a patient who developed graft reinfection despite HBIg prophylaxis after liver transplantation. Sequencing of the HBV genome encoding the surface and polymerase proteins was performed. Changes in the $\mathrm{HBsAg}$ probably explain failure of $\mathrm{HBIg}$ prophylaxis, and associated changes in the viral polymerase may have determined sensitivity to antiviral treatment.

\section{Materials and methods}

HEPATITIS B SEROLOGY

HBsAg was quantitated using the Amerilite enhanced luminescent immunoassay calibrated against the International Standard (supplied by the National Institute for Biological Standards and Control). HBV DNA was quantified by a liquid hybridisation assay (Genostics, Abbott Laboratories, Chicago, USA) and by a quantitative polymerase chain
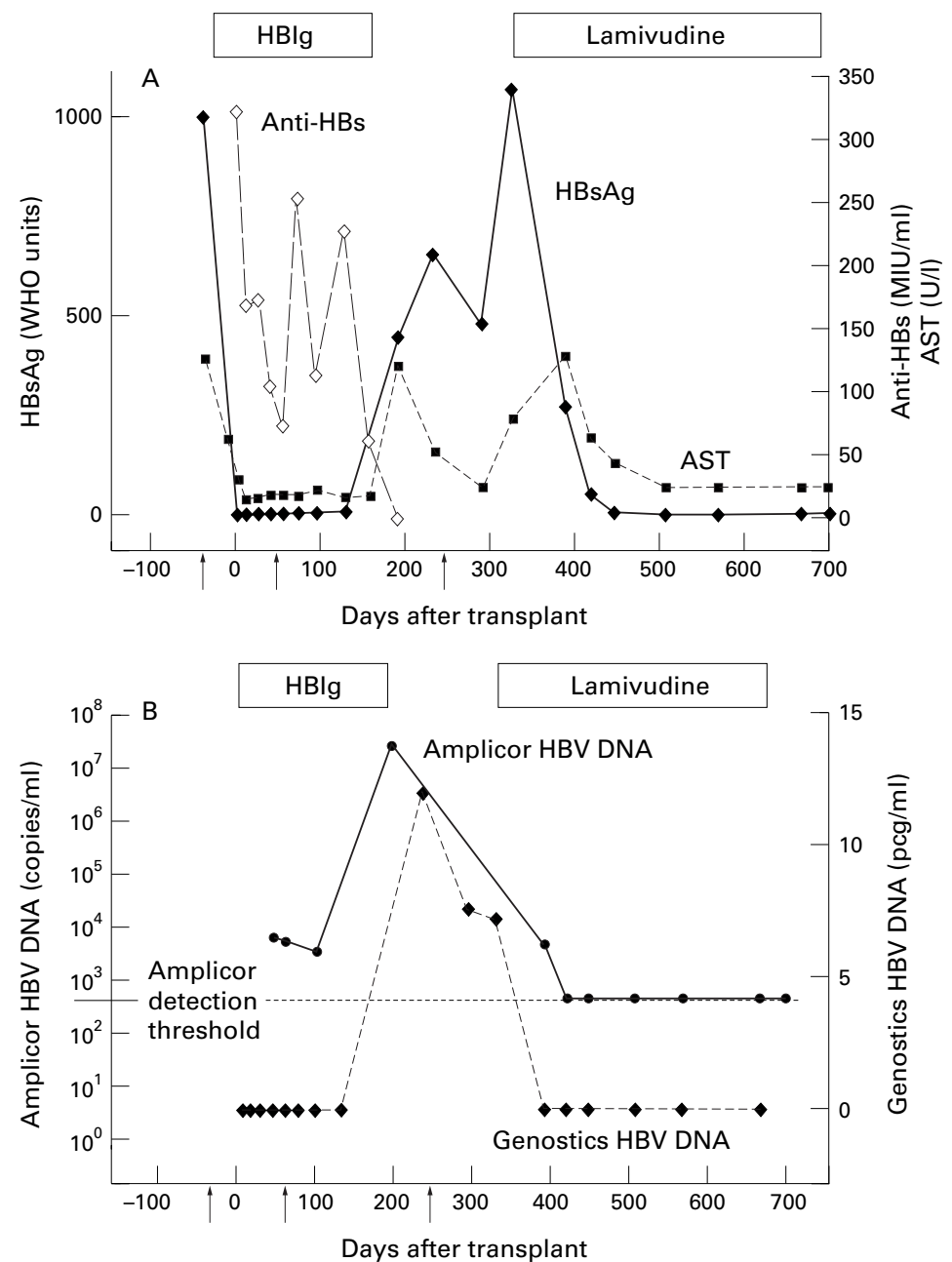

Figure 1 Serum levels of $H B s A g$, anti-HBs, and AST (A) and levels of HBV DNA (B) prior to liver transplantation, during HBIg prophylaxis, and following recurrence of infection. Arrows show time points of HBV surface gene sequence analysis. reaction (PCR; Amplicor HBV Monitor, Roche Molecular Systems, Somerville, New Jersey, USA).

PCR AND SEQUENCE ANALYSIS

Three serum samples were analysed: pretransplant, during prophylaxis, and after recurrence of infection.

DNA was extracted from $50 \mu$ of serum using QIAamp spin columns and amplified by PCR with nested primers flanking the $S$ gene. The 5' primers were: S1: 5'CCTGCTGGT GGCTCCAGTTCC; S6C: 5'GCAGCAA CACGGAAATTCCGAGGACTGGGGACC CTG. The 3' primers were: S2: 5'ATACTT TCCAATCAATAGG; S7D: 5'GACACCAA GCCTTGGTTAAGGGTTTAAATGTAATA ACC.

The entire sequence of the $S$ gene was obtained by direct sequencing of the PCR products with Sequenase PCR product sequencing kit (Amersham, UK) according to the manufacturer's instructions. The sequence of the surface gene prior to transplantation was further examined by cloning the PCR products into plasmid pRK5 and sequencing 11 individual clones.

\section{Case history and results}

A 65 year old white man had established HBV associated cirrhosis with ascites refractory to diuretic treatment. Exposure to HBV may have been at the time of blood transfusion in 1968 .

Initial haematology and biochemistry suggested the presence of end stage liver disease with thrombocytopenia (platelets $68 \times 10^{9} / 1$ ), hyponatraemia $(130 \mathrm{mmol} / \mathrm{l})$, hypoalbuminaemia $(19 \mathrm{~g} / \mathrm{l})$, and coagulopathy (prothrombin time 22/15 seconds), and elevated serum levels of bilirubin $(71 \mu \mathrm{mol} / \mathrm{l})$, alkaline phosphatase (571 U/1), and aspartate aminotransferase (AST; $128 \mathrm{U} / \mathrm{l}$ ). HBsAg was detected at a level of $256 \mathrm{IU} / \mathrm{l}, \mathrm{HbeAg}$ negative and anti-HBe positive. HBV DNA was undetectable by the Genostics assay. Serology for delta virus and hepatitis $\mathrm{C}$ virus was negative.

Liver biopsy confirmed the presence of cirrhosis and immunostains for $\mathrm{HBsAg}$ and $\mathrm{HBcAg}$ were positive. Liver transplantation was performed without complication. Immunosuppression included cyclosporin, azathioprine, and corticosteroids. Biopsy confirmed the presence of acute rejection one week postliver transplantation which required high dose corticosteroids. The rejection resolved and the patient was discharged on day 10 post-liver transplantation.

HBIg (10 000 units; Bioproducts Laboratories, Elstree, UK) was administered during the anhepatic phase of the transplant operation. Subsequently, HBIg (5000 units) was given on each of the first three postoperative days. Thereafter, serum anti-HBs was measured monthly, and further HBIg was given when the titre declined below $100 \mathrm{mIU} / \mathrm{ml}$. At the time of discharge, anti-HBs titre was $323 \mathrm{mIU} / \mathrm{ml}$.

Figure 1 shows subsequent HBV serology, serum AST, and HBV DNA. For five months after liver transplantation, serum was HBsAg negative and anti-HBs titre was maintained at 
Figure 2 (A) Hepatitis $B$ core $(H B c)$ staining of liver biopsy taken 10 months post-liver transplantation, following the development of allograft reinfection. Portal tract contains a moderately dense infiltrate of lymphocytes. There is widespread nuclear and cytoplasmic

immunostaining for $\mathrm{HBc} \mathrm{Ag}$ (immunoperoxidase stain). (B) Biopsy 16 months post-liver transplantation and after four months of lamivudine therapy (100 mg daily). Immunostaining for $\mathrm{HBcAg}$ is now restricted to the nuclei of occasional hepatocytes ( $\mathrm{cAg}$ ). Little inflammatory activity is present.

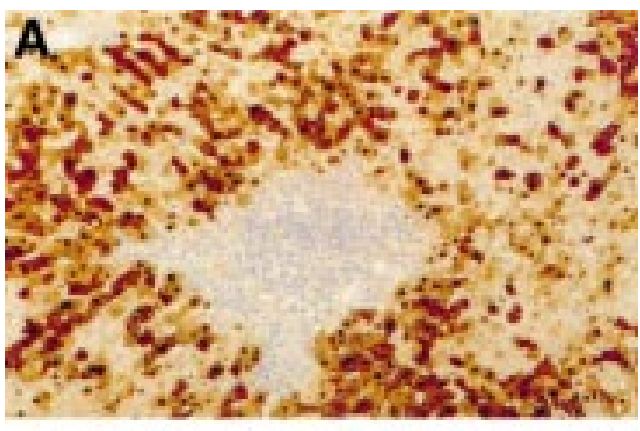

relapse, and became deranged at the time of reappearance of serum HBsAg. Liver biopsy 10 months after liver transplantation confirmed graft reinfection, with chronic hepatitis, moderate periportal inflammatory activity, and mild spotty periseptal inflammation. There was widespread nuclear and cytoplasmic immunostaining for $\mathrm{HBcAg}$ (fig 2A) and focal cytoplasmic immunoreactivity for $\mathrm{HBsAg}$.

Sequential sera were examined to confirm the hypothesis that HBIg failure was associated with HBsAg "escape" mutation. The surface gene sequence pretransplant corresponded to subtype adw, except for a mixture of T and $\mathrm{G}$ at nucleotide 630 (fig 3). This was further examined by cloning the PCR products into plasmid pRK5 and sequencing 11 individual clones; eight were T630 and three G630. The sequence during HBIg prophylaxis was entirely T630. The sequence after HBIg escape and HBsAg recurrence following liver transplantation was homogenous for G630, and also had a change from $\mathrm{A}$ to $\mathrm{C}$ at nucleotide 431 (A431C).

A431C resulted in a replacement of aspartic acid (D) by alanine (A) at amino acid position 144 within the MHR (surfaceD144A). Nucleotide change T630G resulted in switch from serine to arginine at amino acid position 210 (surfaceS210R) outside the MHR. This latter mutation was associated with a change in the polymerase protein (polymeraseS372A).

To treat graft reinfection, lamivudine (100 $\mathrm{mg}$ /day) was commenced 12 months after liver transplantation (supplied for compassionate use by GlaxoWellcome). Prompt suppression of viral replication was observed; HBV DNA prophylaxis was therefore abandoned. Liver function tests were normal before HBV

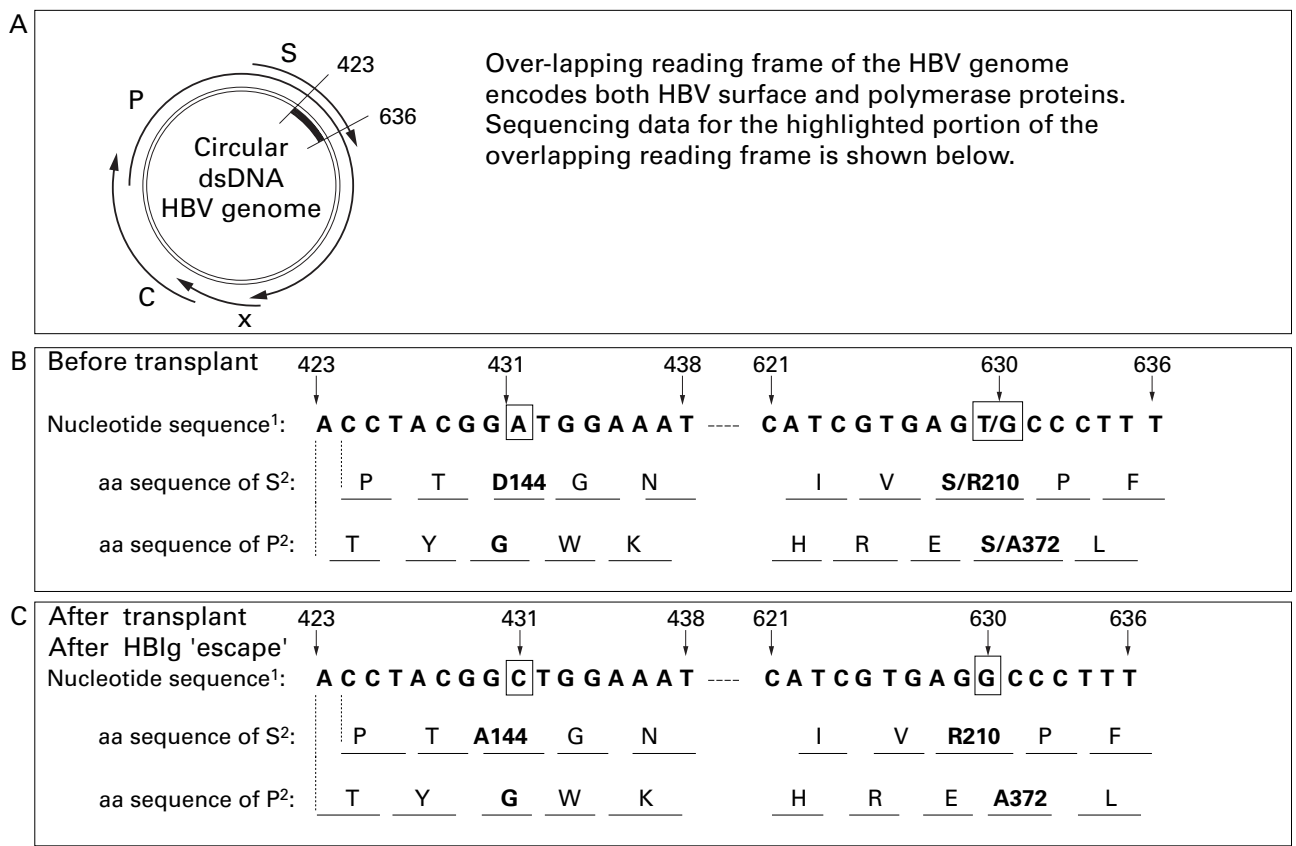

1 The nucleotides are numbered from the start of the $S$ gene

2 The amino acids (aa) are numbered from the start of each protein.

Figure 3 (A) The HBV genome encodes four proteins (core $(C)$, surface $(S)$, polymerase $(P)$, and $X$ proteins). The overlapping reading frame nature of the $H B V$ genome allows a mutation within the genome encoding $S$ and $P$ proteins potentially to alter the amino acid sequence of the $S$ and P proteins. Sequence data show nucleotide and amino acid sequences of the hepatitis B surface gene (nucleotides 423-438 and 621-636) and the corresponding sequence of HBV DNA polymerase: (B) before liver transplantation and (C) after "viral escape" from hepatitis B immunoglobulin prophylaxis. 
became undetectable by the Genostics assay, then undetectable by the Amplicor assay (detection threshold 400 genomic copies $/ \mathrm{ml}$ ). This was associated with $\mathrm{HBeAg}$ and $\mathrm{HBsAg}$ clearance from serum. Liver function tests returned to normal. Liver biopsy 16 months after liver transplantation showed less severe periportal and periseptal inflammation. Immunoreactivity for $\mathrm{HBcAg}$ was considerably less extensive and mainly confined to hepatocyte nuclei (fig 2B). No immunoreactivity for HBsAg was seen.

\section{Discussion}

We report a case of recurrent HBV infection after liver transplantation, occurring despite HBIg prophylaxis. Although there was apparent success of HBIg prophylaxis given during the first five post-transplant months, the application of sensitive qualitative and quantitative PCR assays confirmed persistent viral infection. The subsequent appearance of HBsAg (despite high serum levels of antiHBs) at the time of recurrent hepatitis was consistent with the emergence of a viral escape mutant. Escape mutations are well described in the literature in association with the use of hepatitis B immunoglobulin prophylaxis following liver transplantation but have not been identified in hepatitis B infected patients that have been transplanted without HBIg prophlaxis. ${ }^{15} \mathrm{Se}-$ quential sera were examined and two nucleotide changes in the HBsAg encoding (S) gene were identified. A431C led to an amino acid substitution at position 144 from D to A (surface D144A), which is within the neutralising epitope cluster. In vitro expression ${ }^{16}$ showed the HBsAg had reduced binding in commercial HBsAg assays, consistent with its description as an HBIg escape mutation. As the polymerase encoding $(\mathrm{P})$ gene completely overlaps the $S$ gene, the $S$ gene mutation is always associated with mutation in the $\mathrm{P}$ gene. In this case, because of redundancy of the genetic code, A431C had no effect on the polymerase amino acid sequence. However, the second nucleotide change (T630G) was associated with an amino acid substitution outside the MHR (surfaceS210R) and with a change in the polymerase protein (polymeraseS372A).

It is possible that changes observed in the polymerase protein associated with immune pressure exerted on the HBsAg MHR by HBIg might be associated with important functional changes. For instance, low levels of viral replication (DNA just detectable by the Genostics assay) were observed for this patient (after relapse but before lamivudine) despite concurrent immunosuppressive treatment. Frequently, high levels of replication are observed in this clinical setting. Furthermore, the HBV exhibited remarkable sensitivity to treatment with lamivudine and it is possible that polymerase changes directly affected sensitivity to antiviral treatment. HBV DNA titre declined by $6 \log _{10}$ to become undetectable by PCR, and HBsAg was cleared from the serum. In contrast, in the non- immunosuppressed, lamivudine reduces viral titre by $3-4 \log _{10}$, before the titre plateaus, and HBsAg clearance is rarely observed. ${ }^{12}$ In other words, use of HBIg may result in selection of a virus with surface antigen that has reduced affinity for anti-HBs. The accompanying change in the polymerase protein may be associated with diminished "fitness" compared with the original wild type species. Such polymerase changes may affect the sensitivity and resistance to nucleoside analogues.

This is the first paper to report mutation in the surface gene leading to an alteration in the polymerase gene and its polymerase product in the setting of hepatitis B reinfection following liver transplantation. In view of increasingly complex strategies now being employed to prevent liver graft reinfection, including the use of combination HBIg and lamivudine prior to and following liver transplantation, ${ }^{14}$ it will be important to understand the interactions between $\mathrm{HBIg}$ and HBsAg, with associated changes in the $\mathrm{S}$ and $\mathrm{P}$ genes. The effect of polymerase mutations on clinical course following liver transplantation and the response to antiviral drugs will need to be determined in future studies.

We would like to acknowledge the help of Dr Betty Dragon, Dr Scott Barmat, and Dr Karen Gutekunst at Roche Molecular Systems for performing the quantitative PCR analyses.

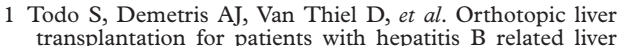
transplantation for patients with hepa
disease. Hepatology 1991;13:619-26.

2 Samuel D, Muller R, Alexander G, et al. Liver transplantation in European patients with the hepatitis B surface antigen. N Engl f Med 1993;329:1842-7.

3 Chen Y-CJ, Delbrook K, Dealwis C, et al. Discontinuous epitopes of hepatitis B surface antigen derived from a filamentous phage peptide library. Proc Natl Acad Sci USA 1996;93:1997-2001.

4 Carman WF, Zanetti AR, Karayiannis P. Vaccine induced escape mutant of hepatitis B virus. Lancet 1990;336:L325-

5 Hawkins AE, Gilson RJ, Gilbert N, et al. Hepatitis B virus surface mutations associated with infection after liver transplantation. $\mathcal{F}$ Hepatol 1996;24:8-14.

6 Carman WF, Trautwein C, Vandeusen FJ, et al. Hepatitis B virus envelope variation after transplantation with and virus envelope variation after transplantation with and
without hepatitis B immune globulin prophylaxis. Hepatology 1996;24:4889-93.

7 Wallace LA, Carman WF. The clinical significance of Wallace LA, Carman WF. The clinical significance of
surface gene variants. Viral Hepatitis Reviews 1997;3:5-16. 8 Marcellin P, Samuel D, Loriot MA, et al. Antiviral effect of adenine arabinoside monophosphate in patients with recurrence of hepatitis B virus infection after liver transplantation. Hepatology 1990;12:S966

9 Gish RG, Lau JYN, Brooks L, et al. Ganciclovir treatment of hepatitis B virus infection in liver transplant recipients. Hepatology 1996;213:1-7.

10 Perillo R, Rakela J, Martin P, et al. Lamivudine for hepatitis B after liver transplantation. Hepatology 1996;24:S182A.

11 Markowitz J, Pakrasi A, Hollis P, et al. Efficacy of lamivudine for prophylaxis and treatment of hepatitis $\mathrm{B}$ in liver transplant patients [abstract]. Hepatology 1996;24:S1872A.

12 Mutimer DJ, Tang H, Dragon B, et al. Lamivudine treatment for hepatitis B virus infected patients undergoing iver transplantation: monitoring response with a quantitative polymerase chain reaction assay [abstract]. Gastroenterology 1997;112:S1342A.

13 Perillo R, Rakela J, Martin P, et al. Long term lamivudine therapy of patients with recurrent hepatitis B post liver transplantation [abstract]. Hepatology 1997;26:S177A.

14 Markowitz JS, Martin P, Conrad AJ, et al. Prophylaxis against hepatitis B recurrence following liver transplantation using combination lamivudine and hepatitis B immune globulin. Hepatology 1998;28:585-9.

15 Carman WF, Trautwein C, Van Deursen. Hepatitis B virus envelope variation after transplantation with and without hepatitis B immune globulin prophylaxis. Hepatology 1996; 24:489-93.

16 Carman WF, Owsianka A, Wallace LA, et al. Antigenic characterisation of pre- and post- liver transplant hepatitis B surface antigen sequences from patients treated with hepatitis B immunoglobulin. F Hepatol (in press). 